The Annals of Applied Probability

1996, Vol. 6, No. 3, 903-921

\title{
A GENERAL STOCHASTIC MODEL FOR NUCLEATION AND LINEAR GROWTH
}

\author{
By L. Holst, ${ }^{1}$ M. P. QUINE AND J. RobINSON \\ Royal Institute of Technology, Stockholm, University of Sydney \\ and University of Sydney
}

\begin{abstract}
The model considered here has arisen in a number of completely separate contexts: release of neurotransmitter at neuromuscular synapses, unravelling of strands of DNA, differentiation of cells into heterocysts in algae and growth of crystals. After a shear transformation the model becomes a Markov process, based on a Poisson process on the upper half plane, homogeneous in the horizontal (time) direction, which increases at unit rate except for occasional "drops." By considering the process separately when it is above or below a given "level," for any interval on the time axis, we obtain in particular exact moment results and prove asymptotic normality for long time intervals for the number of downcrossings in the interval, the total time in the interval when the process is below the specified level and the number of drops in the interval. Limit distributions as the length of interval tends to infinity are obtained for the level at which the interval is "covered." It is shown that several problems considered in the literature have analytic solutions as special cases of the general model. The numerical results from one special case are compared to statistics obtained from experimental data from neurobiology.
\end{abstract}

1. Introduction. The stochastic model considered here has arisen in a number of completely separate contexts: release of neurotransmitter at neuromuscular synapses, unravelling of strands of DNA, differentiation of cells into heterocysts in algae and growth of crystals. The terminal of a neuronal axon at the neuromuscular junction has branches consisting of strands containing many sites from each of which the release of a quantum of transmitter can be evoked by an action potential [Bennett and Robinson (1990)]. Experimental observations of the effects of these releases on the membrane potential of the muscle cell can be obtained both intracellularly, to measure total release, or extracellularly, to measure the time course of individual releases over a section of a branch of the nerve terminal. The releases are found not to follow a Poisson distribution and it has been postulated that at each release an inhibitory substance is simultaneously released from a site, spreading along the terminal branch and preventing releases in regions close to the site where release occurred. Two models for unravelling of DNA strands are discussed in Vanderbei and Shepp (1988) and in Cowan, Chiu and Holst (1995). In these, random sites and times or random sites followed by a random delay time are

Received May 1995; revised March 1996.

${ }^{1}$ Research supported by Australian Research Council Institutional Grant.

AMS 1991 subject classifications. Primary 60J25, 60J15, 60K15, 60K40.

Key words and phrases. Synapses, DNA, cell biology, crystal aggregates, nucleation, inhibition, shear transformation, piecewise deterministic Markov processes. 
the starting points and times of the strand's unravelling, assumed to occur at a fixed rate. The distribution of heterocysts on strands of filamentous blue-green algae is investigated in Wolk (1975). Here cells differentiating into heterocysts inhibit nearby cells from so differentiating. Meijering (1953) considered some models for the formation of crystals, one of which assumed random nucleation followed by growth at a fixed rate. The one-dimensional version of this process is considered here.

In the first example, observations on the number of releases and the time between releases were given and were studied in Bennett and Robinson (1990). An analytic study of the number of releases for a particular simplified model was given in Quine and Robinson (1990). A generalization of this work to include processes inhomogeneous in time is needed to give more realistic models for these experimental results and this is given below. Vanderbei and Shepp (1988) and Cowan, Chiu and Holst (1995) both studied, by rather different means, the time until complete separation of DNA strands under two models. The general model considered in this paper incorporates both these models as special cases and we indicate how to obtain their results. In the third example, attention was focussed on the distances between heterocysts as given by a particular simple model for the inhibitory process. This will be examined under a general model. In the fourth example, lengths of crystals were of interest and these also are examined under the general model. In all cases, it is of interest to examine the properties of the number of initiated points, the distances between them, both at the end of the process (at infinite time) and at some intermediate times, when it is also of interest to look at the number and length of gaps (and of covered regions), and the distribution of times to initiation of the process at various points.

Consider a two-dimensional Poisson process $\Xi$, on time and level, $(t, x)$, homogeneous in time, with rate function $\lambda(x), x>0,-\infty<t<\infty$. This notation is used in line with the convention in point processes, although in each of the applications, "time" actually represents position on a strand and "level" represents time after commencement of the process. This model incorporates as special cases the homogeneous process, where $\lambda(x)=\lambda$, a constant, considered in Vanderbei and Shepp (1988) and the process with $\lambda(x)=(\gamma / \mu) e^{-x / \mu}$ of Cowan, Chiu and Holst (1995). Associated with each point of the process $\Xi$ is a half-line or ray of slope 1 . We will study the process $\left\{X_{t},-\infty<t<\infty\right\}$, defined as the infimum of these rays, as in Figure 1.

In the problems described above it was assumed that there was spread from each position at a constant rate $v$ in both directions. However, because the process is homogeneous in time, $v$ can be taken as $\frac{1}{2}$ by a change of scale and further the distribution is unchanged under the shear transformation $(t, x) \rightarrow(t+x / 2, x)$ [cf. Vanderbei and Shepp (1988)]. Thus questions concerning each of the applications can be transformed into questions concerning the process $\left\{X_{t}\right\}$.

The rest of this paper is set out as follows. In Section 2 we formally define the process $X_{t}$ and consider conditions on the rate $\lambda(x)$ needed for the remainder of the paper. We then look at times when the process first crosses 


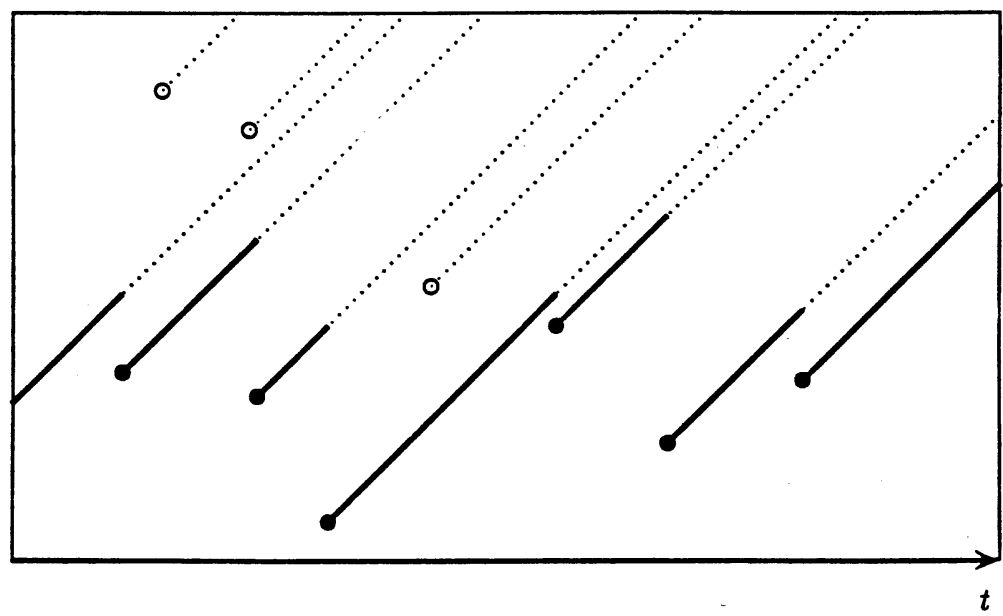

FIG. 1. The process $\left\{X_{t}\right\}$.

a specified level and look at limit results for high levels, thus generalizing the results of Vanderbei and Shepp (1988) and Cowan, Chiu and Holst (1995). In Section 3 we examine the blocks defined by the process's crossing of a fixed level. This enables us to partition the process into a series of independent pieces. We then obtain results on the duration of these blocks, the number of downcrossings in any fixed interval and the number of points of the original process in a block below the specified level. Section 4 contains results concerning the number of drops, the heights and distances between these drops and gives the Markovian properties of these sequences. We obtain the properties of the number of drops in an interval and in each block. In Section 5 we give applications of the results to a number of particular cases considered in the literature.

2. The process $\left\{\boldsymbol{X}_{t}\right\}$. In this section we consider the distribution of $X_{t}$ and the time for $X_{t}$ to reach a specific (possibly high) level. The results are used to derive asymptotic results for the level at which complete coverage of a long interval $(0, t)$ is attained which, in the DNA application and the models for differentiation of cells into heterocysts and crystal growth, is the time until the process is completed.

2.1. Assumptions and the distribution of $X_{t}$. We use throughout the probability space $(\Omega, \mathscr{T}, \mathbf{P})$ defined by the Poisson point process $\Xi$ in the upper half-plane $\{(t, x):-\infty<t<\infty, x>0\}$ with mean measure $\lambda(x) d t d x$. We assume that $\lambda(\cdot)$ is continuous, except for at most a finite number of points, and that for all real numbers $x>0$,

$$
0<\Lambda(x):=\int_{0}^{x} \lambda(y) d y<\infty .
$$


Let $\mathscr{T}_{t}$ be the $\sigma$-algebra generated by this process up to and including time $t$. A filtration is defined by $\left\{\mathscr{F}_{t},-\infty<t<\infty\right\}$. We can describe the process $\left\{X_{t},-\infty<t<\infty\right\}$ as follows. It consists of uniform upward motion at unit speed with occasional jumps downward. The jump rate is $\Lambda\left(X_{t-}\right)$ and after a jump, the new position $X_{t}$ is distributed on the interval $\left(0, X_{t-}\right)$ with the density

$$
\lambda(y) / \Lambda\left(X_{t-}\right) .
$$

Clearly, $\left\{X_{t}\right\}$ is a stationary Markov process with respect to the above filtration.

The distribution of $X_{t}$ at any specific time $t$ can be obtained as follows. First note that the event $\left\{X_{t-} \geq x\right\}$ is the same as the event that there are no points of $\Xi$ in the triangle with corners $(t-x, 0),(t, 0),(t, x)$. As the number of points within the triangle has a Poisson distribution with mean

$$
\Delta(x):=\int_{0}^{x} \Lambda(s) d s, \quad x>0,
$$

we get

$$
\mathbf{P}\left(X_{t} \geq x\right)=e^{-\Delta(x)} .
$$

If we define

$$
\Delta(x)=0, \quad x \leq 0,
$$

then in a similar way we obtain for $t>0$ the conditional distribution

$$
\begin{aligned}
\mathbf{P}\left(X_{t} \geq y \mid X_{0}=x, \mathscr{F}_{0}\right) & =\mathbf{P}\left(X_{t} \geq y \mid X_{0}=x\right) \\
& =\exp (-\Delta(y)+\Delta(y-t)), \quad 0 \leq y \leq x+t .
\end{aligned}
$$

Note that the distribution has an atom at $y=x+t$, corresponding to no jump in the process in $(0, t]$.

We will now make assumptions on the mean measure. First recall l'Hôpital's rule: if $f$ and $g$ are defined and differentiable with $g^{\prime}(x) \neq 0$ for all sufficiently large $x$ and $g(x) \rightarrow \infty$ as $x \rightarrow \infty$, then

$$
\lim _{x \rightarrow \infty} \frac{f(x)}{g(x)}=\lim _{x \rightarrow \infty} \frac{f^{\prime}(x)}{g^{\prime}(x)},
$$

provided the last limit exists (and is finite). We separate three different cases depending on the behavior of $\Lambda(x)$ at infinity.

CASE $\Lambda_{1}$. Assume $\gamma:=\lim _{x \rightarrow \infty} \Lambda(x)<\infty$. In this case the point process $\Xi$ can be generated by a Poisson process with intensity $\gamma$ on the $t$-axis and to each point of this a level is generated (i.i.d.) by the distribution $\Lambda(\cdot) / \gamma$. It follows by l'Hôpital's rule that

$$
\lim _{x \rightarrow \infty} \frac{\Delta(x)}{\gamma x}=\lim _{x \rightarrow \infty} \frac{\Lambda(x)}{\gamma}=1 .
$$


Hence the distribution of $X_{t}$ has an exponentially decreasing tail

$$
e^{-(\gamma+\varepsilon) x}<\mathbf{P}\left(X_{t} \geq x\right)=e^{-\Delta(x)}<e^{-(\gamma-\varepsilon) x}
$$

for $\varepsilon>0$ and all sufficiently large $x$.

CASE $\Lambda_{2}$. Assume $\Lambda(x) \rightarrow \infty$ and $x \lambda(x) / \Lambda(x) \rightarrow \rho$ with $0 \leq \rho<\infty$ as $x \rightarrow \infty$. Then

$$
\lim _{x \rightarrow \infty} \frac{\Delta(x)}{x \Lambda(x)}=\lim _{x \rightarrow \infty} \frac{\Lambda(x)}{\Lambda(x)+x \lambda(x)}=\frac{1}{1+\rho} .
$$

We get for $\varepsilon>0$ and large $x$ that

$$
-x \Lambda(x)\left(\frac{1}{1+\rho}+\varepsilon\right)<\log \mathbf{P}\left(X_{t} \geq x\right)=-\Delta(x)<-x \Lambda(x)\left(\frac{1}{1+\rho}-\varepsilon\right) .
$$

That is, the tail decreases faster than exponentially.

CASE $\Lambda_{3}$. Assume $\Lambda(x) \rightarrow \infty$ and $\lambda(x) / \Lambda(x) \rightarrow c$ with $0<c<\infty$. Then

$$
\lim _{x \rightarrow \infty} \frac{\Delta(x)}{\Lambda(x)}=\lim _{x \rightarrow \infty} \frac{\Lambda(x)}{\lambda(x)}=\frac{1}{c} .
$$

Hence for $\varepsilon>0$ and large $x$,

$$
(c-\varepsilon) x<\log \Lambda(x)<(c+\varepsilon) x .
$$

The tail decreases extremely quickly:

$$
\frac{-e^{(c+\varepsilon) x}}{c+\varepsilon}<\log \mathbf{P}\left(X_{t} \geq x\right)=-\Delta(x)<\frac{-e^{(c-\varepsilon) x}}{c-\varepsilon} .
$$

We think that Cases $\Lambda_{1}-\Lambda_{3}$ cover most situations of interest. The assumptions on the mean measure will imply that all the integrals given below will be finite. It is easily seen that Case $\Lambda_{1}$ implies that the Laplace transform $\mathbf{E}\left(e^{-\alpha X_{t}}\right)$ is finite for $\alpha>-c$ for some $c>0$; Cases $\Lambda_{2}$ and $\Lambda_{3}$ imply that the Laplace transform is finite for all $\alpha$. Thus all moments of $X_{t}$ are finite and we have, for example,

$$
\mathbf{E}\left(X_{t}\right)=\int_{0}^{\infty} e^{-\Delta(x)} d x<\infty .
$$

2.2. Time for $\left\{X_{t}\right\}$ to reach a specific level. Conditional on $X_{0}=x$, consider the random time $\tau$ when the process first hits the level $z>x$ and its Laplace transform

$$
f(x, \alpha)=\mathbf{E}_{x}\left(e^{-\alpha \tau}\right) .
$$

The notations $\mathbf{E}_{x}$ and $\mathbf{P}_{x}$ stand for the conditional expectation and probability given $X_{0}=x$, respectively.

It is easy to derive a recursive relation for the Laplace transform. With probability $\exp (-\Delta(z)+\Delta(x))$ there are no downward jumps in the process before it hits the level $z$; otherwise there is a downward jump before $t=z-x$ 
and after that the process "regenerates" but with another starting value, $y$ say. Taking into account the distribution of the height after such a jump, we get the following recursive relation for $f(x):=f(x, \alpha)$ :

$$
\begin{aligned}
f(x)= & \exp (-\alpha(z-x)) \exp (-\Delta(z)+\Delta(x)) \\
& +\int_{s=0}^{z-x} \int_{y=0}^{s+x} e^{-\alpha s} f(y) \frac{\lambda(y)}{\Lambda(s+x)} \Lambda(s+x) \exp (-\Delta(x+s)+\Delta(x)) d y d s
\end{aligned}
$$

with the boundary condition $f(z)=1$. Differentiating, we obtain

$$
(\alpha+\Lambda(x)) f(x)-f^{\prime}(x)=\int_{0}^{x} f(y) \lambda(y) d y
$$

with $f^{\prime}(0)=\alpha f(0)$, and then after another differentiation,

$$
f^{\prime \prime}(x)=(\alpha+\Lambda(x)) f^{\prime}(x) .
$$

Solving this equation we find

$$
\mathbf{E}_{x}\left(e^{-\alpha \tau}\right)=f(x)=\frac{1+\alpha \int_{0}^{x} \exp (\alpha u+\Delta(u)) d u}{1+\alpha \int_{0}^{z} \exp (\alpha u+\Delta(u)) d u} .
$$

By inverting the Laplace transform, the distribution of $\tau$ can be obtained in any specific case. Differentiation gives

$$
\mathbf{E}_{x}(\tau)=\int_{x}^{z} e^{\Delta(u)} d u
$$

We could also consider $\zeta$, defined to be the time until $\left\{X_{t}\right\}$ drops below level $z$ when $X_{0}=x>z$. This is just the same as the time until the first point of $\Xi$ occurs in the $\operatorname{strip}\{(s, y): s>0,0<y<z\}$. Projecting the points in this strip onto the time axis gives a Poisson process with intensity $\Lambda(z)$. Thus $\zeta$ has an exponential distribution with mean $1 / \Lambda(z)$. That is,

$$
\mathbf{P}(\zeta>t)=e^{-\Lambda(z) t}, \quad t>0 .
$$

2.3. Time for $\left\{X_{t}\right\}$ to reach a high level. It is of interest to study the time until $\left\{X_{t}\right\}$ hits an extreme level. For a high level this is done in Vanderbei and Shepp (1988) for the homogenous case $\lambda(x)=\lambda$, a constant, and by Cowan, Chiu and Holst (1995) both for the homogenous case and for $\lambda(x)=(\gamma / \mu) e^{-x / \mu}$.

From the formula for $\mathbf{E}_{x}(\tau)$ in the previous section and under any of the assumptions $\Lambda_{1}-\Lambda_{3}$, one can show using l'Hôspital rule that for every fixed $x>0$,

$$
\mathbf{E}_{x}(\tau) \Lambda(z) e^{-\Delta(z)} \rightarrow 1, \quad z \rightarrow \infty
$$

We also get

$$
\lim _{z \rightarrow \infty} z \Lambda(z) e^{-\Delta(z)}=0
$$

Therefore

$$
\Lambda(z) \exp (-\Delta(z)) \int_{0}^{z} \exp \left(\alpha u / \mathbf{E}_{x}(\tau)+\Delta(u)\right) d u \rightarrow 1
$$


as $z \rightarrow \infty$ and hence

$$
\mathbf{E}_{x}\left(\exp \left(\frac{-\alpha \tau}{\mathbf{E}_{x}(\tau)}\right)\right)=\frac{1+\left(\alpha / \mathbf{E}_{x}(\tau)\right) \int_{0}^{x} \exp \left(\alpha u / \mathbf{E}_{x}(\tau)+\Delta(u)\right) d u}{1+\left(\alpha / \mathbf{E}_{x}(\tau)\right) \int_{0}^{z} \exp \left(\alpha u / \mathbf{E}_{x}(\tau)+\Delta(u)\right) d u} \rightarrow \frac{1}{1+\alpha} .
$$

Here $1 /(1+\alpha)$ is the Laplace transform of an exponential distribution with unit mean. Therefore as $z \rightarrow \infty$ we have

$$
\mathbf{P}_{x}\left(\max _{0<s<t} X_{s}<z\right)=\mathbf{P}_{x}(\tau>t) \rightarrow \exp \left(-e^{-u}\right)
$$

for fixed $u$ and $t / \mathbf{E}_{x}(\tau) \rightarrow e^{-u}$ or equivalently,

$$
\Delta(z)-\log \Lambda(z)=\log t+u+o(1) .
$$

Note that if $T_{t}$ denotes the level at which the interval $(0, t)$ is completely covered, we have

$$
\mathbf{P}_{x}\left(T_{t}<z\right)=\mathbf{P}_{x}\left(\max _{0<s<t} X_{s}<z\right) .
$$

In the remainder of this section, this result will be used to get asymptotic results on the level at which complete coverage of a long interval $(0, t)$ occurs in a number of special cases.

Consider first the homogenous case for which (2.2) becomes

$$
\lambda z^{2} / 2-\log \lambda z=\log t+u+o(1),
$$

which for $t \rightarrow \infty$ is equivalent to

$$
z=\frac{\log \lambda t^{2}+(1 / 2) \log \log \lambda t^{2}+u+o(1)}{\sqrt{\lambda \log \lambda t^{2}}} .
$$

Hence it follows from (2.1) that, as $t \rightarrow \infty$,

$$
\mathbf{P}_{x}\left(\sqrt{\lambda \log \lambda t^{2}} T_{t}-\log \lambda t^{2}-\frac{1}{2} \log \log \lambda t^{2} \leq u\right) \rightarrow \exp \left(-e^{-u}\right) .
$$

This is proved in a different way by Cowan, Chiu and Holst [(1995), Theorem 2]; it is also obtained in Vanderbei and Shepp (1988), although (as is pointed out) there are some minor gaps in their proof.

Now suppose $\Lambda(x)=\gamma F(x)$, where $F$ is a distribution function with support on $(0, \infty)$ with finite mean $\mu$. This is a special case of $\Lambda_{1}$ in Section 2.1. Formula (2.2) gives

$$
\begin{aligned}
\log \gamma t+u & =-\gamma \int_{0}^{z}(1-F(v)) d v+\gamma z-\log F(z)+o(1) \\
& =\gamma z-\gamma \mu+o(1) .
\end{aligned}
$$

Hence as $t \rightarrow \infty$,

$$
\mathbf{P}_{x}\left(\gamma T_{t}-\log \gamma t-\gamma \mu \leq u\right) \rightarrow \exp \left(-e^{-u}\right) .
$$

This result covers the case considered in Cowan, Chiu and Holst [(1995), Theorem 1] with $\lambda(x)=(\gamma / \mu) e^{-x / \mu}$. 
Let us now consider another special case of $\Lambda_{1}$, where $\Lambda(x)=\gamma F(x)=$ $\gamma(2 / \pi) \arctan x$ so that $F$ has infinite mean. After some calculation we find

$$
\Delta(z)=\gamma\left(z-\frac{2}{\pi}(\log z+1)+o(1)\right)
$$

giving the limit

$$
\mathbf{P}_{x}\left(\gamma T_{t}-\log \gamma t-\frac{2 \gamma}{\pi} \log \log \gamma t-\frac{2 \gamma}{\pi} \leq u\right) \rightarrow \exp \left(-e^{-u}\right),
$$

as $t \rightarrow \infty$.

Another interesting case is $\Lambda(x)=\gamma 2(1-\Phi(1 / \sqrt{x}))$, where $\Lambda_{1}$ of Section 2.1 holds $[\Phi(\cdot)$ is the standard normal distribution function]. This corresponds to a stable distribution with coefficient $\frac{1}{2}$; see Feller (1966). We get

$$
\Delta(z)=\gamma z-\gamma \int_{0}^{z}(2 \Phi(1 / \sqrt{x})-1) d x .
$$

Integration by parts and series expansion gives

$$
\Delta(z)=\gamma(z-\sqrt{8 z / \pi}+1+o(1))
$$

from which we find, as $t \rightarrow \infty$,

$$
\mathbf{P}_{x}\left(\gamma T_{t}-\log \gamma t-\sqrt{\frac{8 \gamma}{\pi} \log \gamma t}+\gamma \leq u\right) \rightarrow \exp \left(-e^{-u}\right) .
$$

For a final example, suppose $\lambda(x)=e^{x}$ so that $\Lambda(x)=e^{x}-1$ and $\Delta(x)=$ $e^{x}-x-1$; this is an example of $\Lambda_{3}$ with $c=1$. We get from (2.2)

$$
e^{z}-2 z-1=\log t+u+o(1)
$$

which gives

$$
\begin{aligned}
z & =\log (\log t+2 \log \log t+u+1) \\
& =\log \log t+(2 \log \log t+u+1) / \log t+o(1 / \log t) .
\end{aligned}
$$

Hence

$$
\mathbf{P}_{x}\left(T_{t} \log t-\log t \log \log t-2 \log \log t-1 \leq u\right) \rightarrow \exp \left(-e^{-u}\right)
$$

as $t \rightarrow \infty$.

3. Blocks. In this section a fixed level $z$ is considered (corresponding to a fixed time in the applications). The behavior of the process is addressed in relation to this level. In the following subsections we consider the distributions of the lengths of these blocks, the number of downcrossings, which is in applications the number of gaps, and the number of points of the original process in each block.

3.1. Duration of blocks. Consider the time points when $\left\{X_{t}\right\}$ crosses the level $z$. These separate the path into "upblocks" where $X_{t}>z$ and "downblocks" with $X_{t}<z$. Because $\left\{X_{t}\right\}$ is Markov, the behavior of the process 
within each block is independent of its behavior in other blocks. The upblocks are probabilistic replicas and so are the downblocks.

By the argument in Section 2.2 we see that the duration $\zeta$ of an upblock is exponential with mean $1 / \Lambda(z)$. To obtain the distribution of the duration $\tau$ of a downblock, note that it begins with a drop from above $z$ to some point in $0<x<z$ chosen according to the density $\lambda(x) / \Lambda(z)$. From the result in Section 2.2 we see that $\tau$ has Laplace transform

$$
\begin{aligned}
\mathbf{E}\left(e^{-\alpha \tau}\right) & =\int_{0}^{z} \frac{\lambda(x)}{\Lambda(z)} \mathbf{E}_{x}\left(e^{-\alpha \tau}\right) d x \\
& =\frac{1+\alpha \int_{x=0}^{z}(\lambda(x) / \Lambda(z)) \int_{u=0}^{x} \exp (\alpha u+\Delta(u)) d u d x}{1+\alpha \int_{0}^{z} \exp (\alpha u+\Delta(u)) d u} \\
& =1-\frac{\alpha}{\Lambda(z)} \frac{\int_{0}^{z} \Lambda(x) \exp (\alpha x+\Delta(x)) d x}{1+\alpha \int_{0}^{z} \exp (\alpha u+\Delta(u)) d u},
\end{aligned}
$$

which determines the distribution of $\tau$. Taking derivatives we find

$$
\mathbf{E}(\tau)=\frac{e^{\Delta(z)}-1}{\Lambda(z)}
$$

and

$$
\operatorname{Var}(\tau)=\frac{2 e^{\Delta(z)}}{\Lambda(z)} \int_{0}^{z}\left(e^{\Delta(u)}-1\right) d u-(\mathbf{E}(\tau))^{2} .
$$

Furthermore, the times for the crossings form an alternating renewal process and results from renewal theory can be used to study different aspects of the process $\left\{X_{t}\right\}$ and also the underlying Poisson point process $\Xi$. For general results on renewal processes see, for example, Asmussen (1987) and Gut (1988).

3.2. Number of downcrossings in an interval. Let us consider $B_{t}$, the number of downcrossings in the interval $(0, t)$. The mean and variance of the duration of a complete block, consisting of one downblock and the succeeding upblock, are

$$
\mu_{B}=\mathbf{E}(\tau+\zeta)=\frac{e^{\Delta(z)}}{\Lambda(z)}
$$

and

$$
\sigma_{B}^{2}=\operatorname{Var}(\tau+\zeta)=\frac{2 e^{\Delta(z)}}{\Lambda(z)} \int_{0}^{z}\left(e^{\Delta(u)}-1\right) d u-\frac{e^{\Delta(z)}\left(e^{\Delta(z)}-2\right)}{\Lambda^{2}(z)} .
$$

As the point process $\Xi$ is stationary, it follows that

$$
\mathbf{E}\left(B_{t}\right)=t / \mu_{B}=t e^{\Delta(z)} / \Lambda(z) .
$$

However, the time point 0 might be chosen in some particular way invalidating stationarity, for example, as the start of a downblock. In any case, Blackwell's 
renewal theorem gives, as $t \rightarrow \infty$,

$$
\mathbf{E}\left(B_{t+h}-B_{t}\right) \rightarrow h / \mu_{B} .
$$

That is, the density of downcrossing is asymptotically

$$
1 / \mu_{B}=\Lambda(z) e^{-\Delta(z)} \text {. }
$$

Furthermore as $t \rightarrow \infty$, well known results of renewal theory give

$$
\operatorname{Var}\left(B_{t}\right)=t \sigma_{B}^{2} / \mu_{B}^{3}+o(t)
$$

and

$$
\frac{B_{t}-\mathbf{E}\left(B_{t}\right)}{\sqrt{\operatorname{Var}\left(B_{t}\right)}} \rightarrow_{d} \mathscr{N}(0,1) .
$$

Another quantity of interest is $C_{t}$, the total time in $(0, t)$ the process spends below level $z$. We have, as $t \rightarrow \infty$,

$$
\frac{C_{t}-t \mathbf{E}(\tau) / \mu_{B}}{\sqrt{t\left(\mathbf{E}(\tau)^{2} \operatorname{Var}(\zeta)+\mathbf{E}(\zeta)^{2} \operatorname{Var}(\tau)\right) / \mu_{B}^{3}}} \rightarrow_{d} \mathscr{N}(0,1)
$$

see, for example, Gut [(1988), page 122].

3.3. Number of points in a downblock. Next consider the points of $\Xi$ in the strip $\{(t, x):-\infty<t<\infty, 0<x<z\}$. Only these points determine the process in downblocks. In upblocks there are no points in this strip. Projecting the points on the time axis gives a Poisson process of intensity $\Lambda(z)$. Mark each such point with 1 if it begins a (down) block; otherwise mark it 0 . The event that a point at $t$, say, has mark 1 is the same as the event that $\Xi$ has no points in the triangle with corners $(t-z, 0),(t, 0),(t, z)$. Hence the probability that the mark is 1 is

$$
p_{1}=e^{-\Delta(z)} \text {. }
$$

The sequence of 1's and 0's generated in this way is evidently a stationary discrete renewal process. Let $\nu$ be the number of points in the Poisson process belonging to the same block, that is, the number of 0 's between successive 1's, plus 1 . Because the times $Z_{1}, Z_{2}, \ldots$ between successive points are independent exponential random variables with mean $1 / \Lambda(z)$, we have

$$
Z_{1}+Z_{2}+\cdots+Z_{\nu}=\tau+\zeta
$$

Now $\nu$ is a stopping time and a well known result of Wald gives

$$
\mathbf{E}(\nu) \mathbf{E}\left(Z_{1}\right)=\mathbf{E}(\tau+\zeta) .
$$

Therefore,

$$
\mathbf{E}(\nu)=\Lambda(z) \frac{e^{\Delta(z)}}{\Lambda(z)}=e^{\Delta(z)} .
$$

Note that

$$
\mathbf{E}(\nu)=e^{\Delta(z)}=1 / p_{1},
$$


which also follows from stationarity. We finally remark that the total number of points in $(0, t) \times(0, z)$ is of course Poisson with mean $t \Lambda(z)$.

4. Drops. Other questions of interest are how many downward jumps or drops, $N_{t}$, there are in $(0, t]$, and the sizes of and distances between such jumps. The number of drops is the number of releases in the neurotransmitter problem and the number of starting points in the other applications. The times at which these occur and the distances between them are used in Section 5.1 to obtain results on growth lengths and nucleation distances; the mean and variance of the number of drops is compared to experimental data in Section 5.2.

4.1. The number of drops in an interval. In this subsection we consider the mean, variance and asymptotic normality of $N_{t}$. Imagine the set $A=$ $\{(s, y): 0 \leq s \leq t, 0 \leq y \leq x+s\}$ split into small elements $d s \times d y$. The event that there is a drop occurring in such an element is the same as the event that there is a point $Q$ of $\Xi$ in the element, which occurs with probability $\lambda(y) d s d y$, and that there are no points in the intersection of the triangle $\Delta(Q)$ with corners $(s, y),(s, 0),(s-y, 0)$ and the first quadrant, which has probability $\exp (-\Delta(y)+\Delta(y-s))$. As the point process $\Xi$ is Poisson, these events are independent and the probability of both occurring is just the product. Summing, that is, integrating, over all elements we obtain the formula

$$
\mathbf{E}_{x}\left(N_{t}\right)=\int_{0}^{t} \int_{0}^{x+s} \lambda(y) \exp (-\Delta(y)+\Delta(y-s)) d y d s .
$$

The above derivation can also be stated in the following more formal way [cf. Mecke (1967), Satz 3.1]. We can write

$$
N_{t}=\int_{A} I(\Xi(\Delta(Q))=0) d \Xi(Q) .
$$

As the point process $\Xi$ is Poisson, we have by independence that

$$
\begin{aligned}
\mathbf{E}_{x}\left(N_{t}\right) & =\int_{A} \mathbf{E}(I(\Xi(\Delta(Q))=0)) \mathbf{E} d \Xi(Q) \\
& =\int_{A} e^{-\Delta(Q)} \lambda(Q) d Q=\int_{0}^{t} \int_{0}^{x+s} \lambda(y) \exp (-\Delta(y)+\Delta(y-s)) d y d s .
\end{aligned}
$$

We think that the above verbal formulation is more illuminating than the formal one. "Verbal formulations" will be used below for similar considerations.

Under stationarity, that is, unconditional on $X_{0}$, using the stationary distribution $\mathbf{P}\left(X_{0} \geq x\right)=e^{-\Delta(x)}$, we get by integrating,

$$
\begin{aligned}
\mathbf{E}_{\mathrm{st}}\left(N_{t}\right) & =t \int_{0}^{\infty} \lambda(y) e^{-\Delta(y)} d y=t \int_{0}^{\infty} \Lambda(y)^{2} e^{-\Delta(y)} d y \\
& =t \mathbf{E}_{\mathrm{st}}\left(\Lambda\left(X_{0}\right)\right) .
\end{aligned}
$$

Next we will derive a formula for the variance, $\operatorname{Var}_{\mathrm{st}}\left(N_{t}\right)$. Split the set $\{(s, y): 0<s<t, 0<y<\infty\}$ into small elements $d s \times d y$. Take two elements with coordinates $\left(0, y_{1}\right)$ and $\left(t u, y_{2}\right), 0<u<1,0<y_{1}, y_{2}<\infty$. The event 
that there are drops in both elements is the same as the event that there is a point in each of the elements and there are no points in the triangles with corners $\left(-y_{1}, 0\right),(0,0),\left(0, y_{1}\right)$, respectively, $\left(t u-y_{2}, 0\right),(t u, 0),\left(t u, y_{2}\right)$. The probability for this is

$$
I\left(y_{1}>y_{2}-t u\right) \lambda\left(y_{1}\right) \lambda\left(y_{2}\right) \exp \left(-\Delta\left(y_{1}\right)\right) \exp \left(-\left(\Delta\left(y_{2}\right)-\Delta\left(y_{2}-t u\right)\right)\right)
$$

times "area elements." Furthermore, the distance between two points taken from a uniform distribution on the unit interval has the density $2(1-u)$ for $0<u<1$.

Using this and symmetry and letting $N_{t}$ be a sum of 1 's and 0 's, each indicating a drop or not in an area element, we find by summing (i.e., integrating) that

$$
\begin{array}{rl}
\mathbf{E}_{\mathrm{st}}\left(N_{t}^{2}\right)=\mathbf{E}_{\mathrm{st}}\left(N_{t}\right)+t^{2} \int_{u=0}^{1} & 2(1-u) \int_{y_{1}=0}^{\infty} \lambda\left(y_{1}\right) \exp \left(-\Delta\left(y_{1}\right)\right) \\
& \times \int_{y_{2}=0}^{y_{1}+t u} \lambda\left(y_{2}\right) \exp \left(-\Delta\left(y_{2}\right)+\Delta\left(y_{2}-t u\right)\right) d y_{2} d y_{1} d u
\end{array}
$$

and, therefore,

$$
\begin{aligned}
\operatorname{Var}_{\mathrm{st}}\left(N_{t}\right)= & t \int_{0}^{\infty} \lambda(y) \exp (-\Delta(y)) d y+t^{2} \int_{y_{1}=0}^{\infty} \lambda\left(y_{1}\right) \exp \left(-\Delta\left(y_{1}\right)\right) \\
& \times \int_{u=0}^{1} 2(1-u) \int_{y_{2}=0}^{y_{1}+t u} \lambda\left(y_{2}\right) \exp \left(-\left(\Delta\left(y_{2}\right)-\Delta\left(y_{2}-t u\right)\right)\right) d y_{2} d u d y_{1} \\
& -t^{2}\left(\int_{0}^{\infty} \lambda(y) \exp (-\Delta(y)) d y\right)^{2} .
\end{aligned}
$$

We therefore find that $\operatorname{Var}_{\mathrm{st}}\left(N_{t}\right) / t$ equals

$$
\begin{aligned}
\int_{0}^{\infty} & \lambda(y) \exp (-\Delta(y)) d y \\
& -\int_{y_{1}=0}^{\infty} \lambda\left(y_{1}\right) \exp \left(-\Delta\left(y_{1}\right)\right) \int_{y_{2}=0}^{\infty} \lambda\left(y_{2}\right) \exp \left(-\Delta\left(y_{2}\right)\right) \int_{u=0}^{t \wedge y_{2}} 2\left(1-\frac{u}{t}\right) d u d y_{2} d y_{1} \\
& +\int_{y_{1}=0}^{\infty} \lambda\left(y_{1}\right) \exp \left(-\Delta\left(y_{1}\right)\right) \\
& \times \int_{y_{2}=0}^{y_{1}} \int_{u=0}^{t} \frac{2}{1-u / t} \lambda\left(y_{2}+u\right) \exp \left(-\left(\Delta\left(y_{2}+u\right)-\Delta\left(y_{2}\right)\right)\right) d u d y_{2} d y_{1} .
\end{aligned}
$$

Hence as $t \rightarrow \infty$,

$$
\begin{aligned}
\operatorname{Var}_{\mathrm{st}}\left(N_{t}\right) / t \rightarrow & \int_{0}^{\infty} \lambda(y) \exp (-\Delta(y)) d y \\
& -\int_{y_{1}=0}^{\infty} \lambda\left(y_{1}\right) \exp \left(-\Delta\left(y_{1}\right)\right) \int_{y_{2}=0}^{\infty} \exp \left(-\Delta\left(y_{2}\right)\right) 2 \lambda\left(y_{2}\right) y_{2} d y_{2} d y_{1} \\
& +\int_{y_{1}=0}^{\infty} \lambda\left(y_{1}\right) \exp \left(-\Delta\left(y_{1}\right)\right) \\
& \times \int_{y_{2}=0}^{y_{1}} \int_{u=y_{2}}^{\infty} 2 \lambda(u) \exp \left(-\left(\Delta(u)-\Delta\left(y_{2}\right)\right)\right) d u d y_{2} d y_{1} .
\end{aligned}
$$


One might also be interested in the distribution of $N_{t}$. For large $t$, asymptotic normality follows by the following argument. Consider for a level $z$ complete blocks, each consisting of a downblock and the succeeding upblock. Because the blocks are independent, the number of drops in the blocks are independent too. As $N_{t}$ is the sum of the drops in the blocks, we can use the results for renewal processes to justify asymptotic normality; see Gut [(1988), Theorems IV 2.3 and IV 2.4]. Thus we have

$$
\frac{N_{t}-\mathbf{E}_{\mathrm{st}}\left(N_{t}\right)}{\sqrt{\operatorname{Var}_{\mathrm{st}}\left(N_{t}\right)}} \rightarrow_{d} \mathscr{N}(0,1)
$$

The same techniques can be used to derive analogous results for $N_{t, z}$, the number of drops in the set $\{(s, y): 0<s<t, 0<y<z\}$.

We finish this section by considering $\mathbf{E}_{\mathrm{st}}\left(N_{t}\right)$ and $\operatorname{Var}_{\mathrm{st}}\left(N_{t}\right)$ in the homogeneous case $[\lambda(x)=\lambda]$. It is easy to show

$$
\mathbf{E}_{\mathrm{st}}\left(N_{t}\right)=t \sqrt{\frac{\pi \lambda}{2}}
$$

and

$$
\begin{aligned}
\frac{\operatorname{Var}_{\mathrm{st}}\left(N_{t}\right)}{t} \rightarrow & \int_{0}^{\infty} \lambda \exp \left(-\frac{\lambda y^{2}}{2}\right) d y\left(1-2 \int_{0}^{\infty} \lambda y \exp \left(-\frac{\lambda y^{2}}{2}\right) d y\right) \\
& +\int_{y=0}^{\infty} \lambda \exp \left(-\frac{\lambda y^{2}}{2}\right) \\
& \quad \times \int_{x=0}^{y} \exp \left(\frac{\lambda x^{2}}{2}\right) \int_{u=x}^{\infty} 2 \lambda \exp \left(-\frac{\lambda u^{2}}{2}\right) d u d x d y \\
= & \sqrt{\frac{\pi \lambda}{2}}(2 \log 2-1)
\end{aligned}
$$

after some algebra. Equations (4.1) and (4.2) are in accordance with the results of Quine and Robinson [(1990), page 501], which concern linear growth on a finite interval.

4.2. Heights of and distances between drops. The process $\left\{X_{t}, t \geq 0\right\}$ can be described in terms of $H_{0}, T_{0}, H_{1}, T_{1}, H_{2}, T_{2}, \ldots$, where $T_{0}, T_{0}+T_{1}$, $T_{0}+T_{1}+T_{2}, \ldots$ are the times to the first, second, third, ... discontinuity, or drop, in $\left\{X_{t}\right\}$ and $H_{0}, H_{1}, H_{2}, \ldots$ are the heights of $\left\{X_{t}\right\}$ at $0, T_{0}, T_{0}+T_{1}, \ldots$ We will also let $Y_{1}, Y_{2}, \ldots$ denote the predrop heights, so $Y_{1}=X\left(T_{0}-\right)$ and so on; note that $Y_{n}=H_{n-1}+T_{n-1}$. Then the sequences $\left\{H_{1}, H_{2}, \ldots\right\}$ and $\left\{\left(H_{1}, T_{0}\right),\left(H_{2}, T_{1}\right), \ldots\right\}$ are Markov chains with continuous state spaces. It is of particular interest to obtain the transition probabilities and the stationary distributions of $H_{i}, T_{i}$ and $S_{i+1}=H_{i}+T_{i}-H_{i+1}$, the size of the $(i+1)$ th drop.

The conditional density of $\left(H_{i+1}, T_{i}\right)$ given $\left(H_{i}, T_{i-1}\right)$, that is, the transition density for the second chain, is, for $0<y<x+t$,

$$
f_{H_{i+1}, T_{i} \mid H_{i}, T_{i-1}}(y, t \mid x, u)=f_{H_{i+1}, T_{i} \mid H_{i}}(y, t \mid x)=\lambda(y) \exp (-\Delta(x+t)+\Delta(x)),
$$


so the transition probability density from $H_{i}=x$ to $H_{i+1}=y$ is

$$
p(x, y)=f_{H_{i+1} \mid H_{i}}(y \mid x)= \begin{cases}\lambda(y) e^{\Delta(x)} \int_{y}^{\infty} e^{-\Delta(u)} d u, & y>x, \\ \lambda(y) e^{\Delta(x)} \int_{x}^{\infty} e^{-\Delta(u)} d u, & y<x .\end{cases}
$$

Further,

$$
f_{T_{i} \mid H_{i}}(t \mid x)=\Lambda(x+t) \exp (-\Delta(x+t)+\Delta(x))
$$

and for $0<y<x+t$,

$$
f_{T_{i} \mid H_{i}, H_{i+1}}(t \mid x, y)=e^{-\Delta(x+t)} / \int_{x \vee y}^{\infty} e^{-\Delta(u)} d u .
$$

By the usual "empty triangle" argument, the stationary density $f_{H}(y)$ of $H_{i}$ is proportional to $\lambda(y) e^{-\Delta(y)}$. Thus

$$
f_{H}(y)=\kappa \lambda(y) e^{-\Delta(y)},
$$

where

$$
\kappa=1 / \int_{0}^{\infty} \lambda(u) e^{-\Delta(u)} d u .
$$

From this we obtain the stationary distribution of $T_{i}$ from

$$
\begin{aligned}
\mathbf{P}_{\mathrm{st}}\left(T_{i}>t\right) & =\int_{0}^{\infty} f_{H}(x) \exp (-\Delta(x+t)+\Delta(x)) d x \\
& =\kappa \int_{0}^{\infty} \lambda(x) \exp (-\Delta(x+t)) d x .
\end{aligned}
$$

Integration leads to the stationary expectation of $T_{i}$ as

$$
\mathbf{E}_{\mathrm{st}}\left(T_{i}\right)=\int_{0}^{\infty} \mathbf{P}_{\mathrm{st}}\left(T_{i}>t\right) d t=\kappa .
$$

REMARK. Noting that we can write

$$
\mathbf{P}_{\mathrm{st}}\left(H_{i}>h\right)=\kappa \int_{0}^{\infty} \lambda(x+h) e^{-\Delta(x+h)} d x,
$$

it is easy to see that $H_{i}$ and $T_{i}$ have the same stationary distribution if and only if $\lambda(x)=\lambda$, a constant.

We can similarly obtain the following simple formulas for the stationary moments of $H_{i}$ and $T_{i}$ :

$$
\begin{aligned}
\mathbf{E}_{\mathrm{st}}\left(H_{i}\right) & =\kappa \int_{0}^{\infty} x \lambda(x) e^{-\Delta(x)} d x \\
\operatorname{Var}_{\mathrm{st}}\left(H_{i}\right) & =\kappa \int_{0}^{\infty} x^{2} \lambda(x) e^{-\Delta(x)} d x-\left(\kappa \int_{0}^{\infty} x \lambda(x) e^{-\Delta(x)} d x\right)^{2}, \\
\operatorname{Var}_{\mathrm{st}}\left(T_{i}\right) & =2 \kappa \int_{0}^{\infty} \Delta(x) e^{-\Delta(x)} d x-\kappa^{2}
\end{aligned}
$$


and

$$
\operatorname{Cov}_{\mathrm{st}}\left(H_{i}, T_{i}\right)=\kappa \int_{0}^{\infty}(1-\Delta(x)) e^{-\Delta(x)} d x-\kappa^{2} \int_{0}^{\infty} x \lambda(x) e^{-\Delta(x)} d x .
$$

REMARK. In the particular case when $\lambda(x)=\lambda$ is constant, these results give

$$
\operatorname{corr}_{\mathrm{st}}\left(H_{i}, T_{i}\right)=\frac{1 / 2-2 / \pi}{1-2 / \pi} \approx-0.38 .
$$

There are two other related results concerning $H_{0}, T_{0}, H_{1}, T_{1}, \ldots$ A completed left branch of length $l$ in the original unsheared process is transformed to a drop of size $S=2 l$ and a completed right branch of length $r$ is transformed to an interdrop time of duration $T=2 r$. Thus the size of drop, for example, $S_{i+1}=H_{i}+T_{i}-H_{i+1}$, has the same stationary distribution as $T_{i}$.

We might expect that for all $x>0$,

$$
\mathbf{P}_{\text {st }}\left(H_{i}>x\right) \leq \mathbf{P}_{\text {st }}\left(X_{t}>x\right) \leq \mathbf{P}_{\text {st }}\left(H_{i}+T_{i}>x\right) .
$$

In the homogeneous case, $\lambda(x)=\lambda$, a constant, it is not difficult to verify this analytically.

4.3. Markov renewal theory for drops. From the forms for the transition densities in Section 4.2 it can be seen that both Markov chains are aperiodic recurrent Harris chains [see, e.g., Durrett (1991), pages 282 and 287] with stationary distributions. A consequence of this is, for example, that uniformly in $A$ as $n \rightarrow \infty$,

$$
\mathbf{P}_{x}\left(H_{n} \in A\right) \rightarrow \int_{A} f_{H}(y) d y .
$$

Furthermore the Markov chain $\left\{\left(X_{0}, 0\right),\left(H_{1}, T_{0}\right),\left(H_{2}, T_{1}\right), \ldots\right\}$ defines a Markov renewal process, for which ergodic properties can be deduced using Theorem 2.1 in Alsmeyer (1994). However, we think that the following direct approach is of interest as it illuminates some of the results obtained above.

In Section 4.1 we considered the number of drops, $N_{t}$, in $(0, t]$, "renewals" in the above process, and found that under stationarity

$$
\mathbf{E}_{\mathrm{st}}\left(N_{t}\right)=t \int_{0}^{\infty} \lambda(y) e^{-\Delta(y)} d y .
$$

In the previous section it was proved that the mean time between drops is

$$
\mathbf{E}_{\mathrm{st}}\left(T_{i}\right)=1 / \int_{0}^{\infty} \lambda(y) e^{-\Delta(y)} d y,
$$

which gives

$$
\mathbf{E}_{\mathrm{st}}\left(N_{t}\right)=t / \mathbf{E}_{\mathrm{st}}\left(T_{i}\right) .
$$

From the explicit formula for $\mathbf{E}_{x}\left(N_{t}\right)$ it is not difficult to show that

$$
\mathbf{E}_{x}\left(N_{t+h}-N_{t}\right) \rightarrow h / \mathbf{E}_{\mathrm{st}}\left(T_{i}\right), \quad t \rightarrow \infty,
$$

which also follows from the Markov renewal theorem in Alsmeyer (1994). 
4.4. Mean and variance for the number of drops in a block. Let $N_{\zeta}$ be the number of drops in an upblock. To find the mean of $N_{\zeta}$, consider the Poisson process in the triangular region bounded by the lines $x=z$ and $x=z+t$, $t \geq 0$. In order that a small rectangle $(t, t+d t) \times(y, y+d y)$ in this region contributes to $N_{\zeta}$, it is necessary that (a) there is a point there, (b) the point is a drop and (c) $\zeta>t$. Using the fact that $\zeta$ has an exponential distribution and independence, we get

$$
\begin{aligned}
\mathbf{E}\left(N_{\zeta}\right) & =\int_{0}^{\infty} \int_{z}^{z+t} \lambda(y) \exp (-(\Delta(y)-\Delta(z)-(y-z) \Lambda(z))) \exp (-\Lambda(z) t) d t d y \\
& =\frac{e^{\Delta(z)}}{\Lambda(z)} \int_{z}^{\infty} \lambda(y) e^{-\Delta(y)} d y
\end{aligned}
$$

on changing the order of integration. The variance of $N_{\zeta}$ can be found in the same way to be

$$
\operatorname{Var} N_{\zeta}=\mathbf{E} N_{\zeta}+\left(\mathbf{E} N_{\zeta}\right)^{2}+2 I_{2}
$$

where

$$
\begin{aligned}
& I_{2}=e^{\Delta(z)} \iint \lambda\left(y_{2}\right) \exp \left(-\Delta\left(y_{2}\right)\right) \\
& \quad \times \iint \lambda(v-u+z) \exp (-\Delta(v+z-u) \\
& \left.\quad-u \Lambda(z)+\Delta\left(v-t_{2}+y_{2}\right)\right) d u d v d t_{2} d y_{2},
\end{aligned}
$$

the limits being $0<u<t_{2}+z-y_{2}, t_{2}-y_{2}+z<v<t_{2}, z<y_{2}<z+t_{2}$ and $0<t_{2}<\infty$.

Let $N_{\tau}$ be the number of drops in a downblock (including the initial drop). The above method is not suitable for finding the moments of $N_{\tau}$, but it is easy to derive a recursive relation. Conditional on a starting value of $x \in(0, z)$, there is no other drop with probability $\exp (-\Delta(z)+\Delta(x))$, and otherwise there is another drop before $t=z-x$ and the downblock "regenerates." Taking into account the distribution of the height after this drop, we get the following recursive relation for $g(x):=\mathbf{E}_{x}\left(N_{\tau}\right)$ :

$$
g(x)=\exp (-\Delta(z)+\Delta(x))
$$

$$
\begin{aligned}
& +\int_{0}^{z-x} \exp (-\Delta(x+t)+\Delta(x)) \Lambda(x+t) \int_{0}^{x+t}(1+g(y)) \frac{\lambda(y)}{\Lambda(x+t)} d y d t \\
= & 1+\int_{x}^{z} \exp (-\Delta(s)+\Delta(x)) \int_{0}^{s} g(y) \lambda(y) d y d s
\end{aligned}
$$

with the boundary condition $g(z)=1$. Differentiating, we obtain

$$
\begin{gathered}
g^{\prime}(x)=-\int_{0}^{x} g(y) \lambda(y) d y+\Lambda(x)(g(x)-1), \\
g^{\prime \prime}(x)-\Lambda(x) g^{\prime}(x)=-\lambda(x),
\end{gathered}
$$


giving

$$
g(x)=1+\int_{x}^{z} e^{\Delta(y)} \int_{0}^{y} e^{-\Delta(t)} \lambda(t) d t d y .
$$

The initial drop height $x$ has density $\lambda(x) / \Lambda(z)$, so

$$
\begin{aligned}
\mathbf{E} N_{\tau} & =\int_{0}^{z} g(x) \frac{\lambda(x)}{\Lambda(z)} d x \\
& =\frac{e^{\Delta(z)}}{\Lambda(z)} \int_{0}^{z} \lambda(y) e^{-\Delta(y)} d y .
\end{aligned}
$$

The variance of $N_{\tau}$ can be obtained similarly, since it follows as above that

$$
\mathbf{E} N_{\tau}^{2}=1+\frac{1}{\Lambda(z)} \int_{0}^{z}(\exp (\Delta(z)-\Delta(y))-1) \lambda(y)(1+2 g(y)) d y .
$$

5. Applications. It is worthwhile to consider some special cases. First, we will apply the results of earlier sections to give more directly some results of Wolk (1975) and Meijering (1953). Then we apply the results of Section 4.1 to a model proposed in Bennett and Robinson (1990) and obtain numerical results corresponding to some data for number of releases of neurotransmitter at synapses.

5.1. Nucleation distances and growth lengths. In applications, two quantities of interest in the "unsheared" process are the distance between adjacent nucleation sites, $\xi$ say, and the final length of the growth from one such site, $\eta$ say. In terms of the sheared process, by noting that $Y_{2}=H_{1}+T_{1}$, we have

$$
2 \xi=T_{1}+Y_{2}-H_{2} \text { and } 2 \eta=Y_{1}-H_{1}+T_{1} .
$$

We proceed to derive the densities of $\xi$ and $\eta$ in the case $\lambda(x)=\lambda$.

Note first that $2 \xi={ }_{d} U H_{1}+(1+U) T_{1}$, where $U$ is uniform on $(0,1)$, independent of $H_{1}$ and $T_{1}$. The results of Section $4.2 \mathrm{imply}$ that, in general,

$$
f_{H T}(h, t)=\kappa \lambda(h) \Lambda(t+h) e^{-\Delta(t+h)} .
$$

In the homogenous case, $\kappa=\sqrt{2 / \pi \lambda}$ and so

$$
\mathbf{P}\left(u H_{1}+(1+u) T_{1} \leq 2 x\right)=\sqrt{\frac{2}{\pi}} \lambda^{3 / 2} \iint(t+h) \exp \left(-\frac{\lambda(t+h)^{2}}{2}\right) d h d t,
$$

where the integral is over the region $u h+(1+u) t \leq 2 x$. It follows that the density of $\xi$ is

$$
\begin{aligned}
f_{\xi}(x)= & 2 \sqrt{\frac{2 \lambda}{\pi}} \int_{0}^{1}\left(\exp \left(-\frac{2 \lambda x^{2}}{(1+u)^{2}}\right)-\exp \left(\frac{-2 \lambda x^{2}}{u^{2}}\right)\right) d u \\
= & 4 \sqrt{\frac{2 \lambda}{\pi}}\left(\exp \left(\frac{-\lambda x^{2}}{2}\right)-\exp \left(-2 \lambda x^{2}\right)\right) \\
& +8 \lambda x(1-2 \Phi(2 \sqrt{\lambda} x)+\Phi(\sqrt{\lambda} x)),
\end{aligned}
$$


where $\Phi(\cdot)$ is the standard normal distribution function. With $v=1 / 2$, this corresponds to equation (xvi) of Wolk (1975) (there are two obvious misprints in that equation).

To obtain the density of $\eta$ it is necessary to use the fact that $Y_{1}$ and $T_{1}$ are conditionally independent, given $H_{1}$, so that

$$
\mathbf{P}\left(\eta \leq x \mid H_{1}=h\right)=\iint f_{Y \mid H}(y \mid h) f_{T \mid H}(t \mid h) d y d t,
$$

where the integral is over the triangular region $t \geq 0, y \geq h, y-h+t \leq 2 x$. It follows from (5.1) that $Y_{1}={ }_{d} Y_{2}=H_{1}+T_{1}$ has density

$$
f_{Y}(y)=\kappa \Lambda^{2}(y) e^{-\Delta(y)},
$$

so that

$$
f_{Y \mid H}(y \mid h)=\frac{f_{H \mid Y}(h \mid y) f_{Y}(y)}{f_{H}(h)}=\Lambda(y) \exp (\Delta(h)-\Delta(y)), \quad y \geq h .
$$

In the homogeneous case, using (5.2) and (4.3), integrating with respect to $t$ first and putting $z=y-h$ gives, after some calculation,

$$
\begin{aligned}
f_{\eta \mid H}(x \mid h)= & 2 \lambda \exp \left(-\lambda(2 x+h)^{2} / 2+\lambda h^{2} / 2\right) \\
& \times\left(2 x-\int_{0}^{2 x}\left(1-\lambda(z+h)^{2}\right) \exp \left(-\lambda(z-x)^{2}+\lambda x^{2}\right) d z\right)
\end{aligned}
$$

and, putting $w=(z-x) \sqrt{\lambda}$,

$$
\begin{aligned}
f_{\eta \mid H}(x \mid h)= & 2 \lambda \exp (-2 \lambda x(x+h)) \\
& \times\left(x+\sqrt{\pi / \lambda} \exp \left(\lambda x^{2}\right)(2 \Phi(\sqrt{2 \lambda} x)-1)\left(\lambda(h+x)^{2}-1 / 2\right)\right) .
\end{aligned}
$$

Since the density of $H$ is [from (4.5)]

$$
f_{H}(h)=\sqrt{\frac{2 \lambda}{\pi}} \exp \left(-\left(\frac{\lambda}{2}\right) h^{2}\right), \quad h \geq 0,
$$

integration gives

$$
f_{\eta}(x)=2 \sqrt{\lambda}(1-\Phi(2 \sqrt{\lambda} x))\left(2 x \sqrt{\lambda}+\sqrt{\pi}\left(2 \lambda x^{2}+1\right) \exp \left(\lambda x^{2}\right)(2 \Phi(\sqrt{2 \lambda} x)-1)\right) .
$$

This corresponds to (32) of Meijering (1953) with $u=x \sqrt{\lambda}$.

5.2. An example of neurobiological data. Data obtained by Professor M. R. Bennett of the Neurobiology Research Centre at the University of Sydney were reported in Quine and Robinson (1992). For these data the mean and variance of the number of releases from 800 impulses were 1.340 and 0.680 , respectively. A model for the rate of release proposed in Bennett and Robinson (1990) was equivalent to defining

$$
\lambda(x)=\gamma \theta^{k} x^{k-1} e^{-\theta x} / \Gamma(k),
$$


with the particular value $k=5$ suggested. Notice that $\Lambda(\infty)=\gamma$. Estimation of $\gamma, \theta$ and $v$ in a doctoral thesis of Thomson (1994) gave 2.04, 2.94 and 0.20, respectively. These estimates are based only on the times for the first two releases. Simulations based on these parameters using 10,000 trials gave a mean and variance of 1.57 and 1.04, and numerical integration of the results of Section 4.1 using MATLAB gave a mean and variance of 1.54 and 1.00. The results are qualitatively satisfactory, but there are difficulties with the model due to detection errors as pointed out in Thomson (1994) and Thomson, Lavidis, Robinson and Bennett (1995).

\section{REFERENCES}

AlsmeYer, G. (1994). On the Markov renewal theorem. Stochastic Process. Appl. 50 37-56.

Asmussen, S. (1987). Applied Probability and Queues. Wiley, New York.

BenNeTt, M. R. and RoBINson, J. (1990). Probabilistic secretion of quanta from nerve terminals at synaptic sites on muscle cells: non-uniformity, autoinhibition and the binomial hypothesis. Proc. Roy. Soc. London Ser. B 239 329-358.

Cowan, R., CHIU, S. N. and Holst, L. (1995). A limit theorem for the replication time of a DNA molecule. J. Appl. Probab. 32 296-303.

Durrett, R. (1991). Probability: Theory and Examples. Wadsworth \& Brooks-Cole, Belmont, CA.

FELleR, W. (1966). An Introduction to Probability Theory and Its Applications 2, 1st ed. Wiley, New York.

GuT, A. (1988). Stopped Random Walks. Springer, New York.

MECKE, J. (1967). Stationäre zufällige Maße auf lokalkompakten Abelschen Gruppen. Z. Wahrsch. Verw. Gebiete 9 36-58.

MEIJERING, J. L. (1953). Interface area, edge length and number of vertices in crystal aggregates with random nucleation. Philips Res. Ref. 8 270-290.

Quine, M. P. and Robinson, J. (1990). A linear random growth model. J. Appl. Probab. 27499 509.

Quine, M. P. and Robinson, J. (1992). Estimation for a linear growth model. Statist. Probab. Lett. 15 295-297.

Thomson, P. C. (1994). Statistical models of synaptic transmission. Ph.D. dissertation, Univ. Sydney.

Thomson, P. C., Lavidis, N. A., Robinson, J. and Bennett, M. R. (1995). Probabilistic secretion of quanta at somatic motor-nerve terminals: the fusion pore model, quantal detection and autoinhibition. Philos. Trans. Roy. Soc. London Ser. B 349 197-214.

VANDERBEI, R. J. and SHEPP, L. A. (1988). A probabilistic model for the time to unravel a strand of DNA. Comm. Statist. Stochastic Models 4 299-314.

Wolk, C. P. (1975). Formation of one-dimensional patterns by stochastic processes and by filamentous blue-green algae. Developmental Biology 46 370-382.

LARS HOLST

DEPARTMENT OF MATHEMATICS

ROYAL InSTITUTE OF TECHNOLOGY

S-100 44 STOCKHOLM

SWEDEN

E-MAIL: lholst@math.kth.se

\section{MALCOLM QUINE}

JOHN ROBINSON

School of Mathematics and Statistics

UNIVERSITY OF SYDNEY

N. S. W. 2006

Australia

E-MAIL: quine_m@maths.usyd.edu.au robinso_j@maths.usyd.edu.au 